\title{
A PEDRA E O SOFRIMENTO: REPRESENTAÇÕES E HISTORICIZAÇÕES DO HOLOCAUSTO
}

THE STONE AND THE SUFFERING: REPRESENTATIONS AND HISTORICIZATIONS OF THE HOLOCAUST

\section{LA PIERRE ET LA PEINE \\ REPRÉSENTATIONS ET HISTORICISATIONS DE L'HOLOCAUSTE ${ }^{1}$}

Enviado em 8 de agosto de 2015

Resumo: Esse artigo interroga a noção de museu e a relação entre História e memória a partir do difícil trabalho de historicizar os objetos nos museus. Este texto é uma tentativa de estudar os memoriais e os processos de memória sob um ponto de vista antropológico. Os debates que envolvem a representação do holocausto, assim como o trabalho do artista contemporâneo Jochen Gerz, servem de fio condutor a essa reflexão. Procura-se compreender, portanto, em que medida o dever de memória não é uma estratégia de esquecimento. Os memoriais e museus expõem e celebram também o que uma sociedade deve esquecer para existir.

Palavras-chave: Memória. Memoriais. Holocausto. Objetos.

Abstract: This article explores the concept of museum and the relation between history and memory through the difficult task of historicizing objects housed in museums. This text is an attempt to study memorials and the memory process from an anthropological standpoint, focussing on the debates surrounding representations of the Holocaust as well as the contemporary artist Jochen Gerz. The text aims to understand to what extent the imperative of memory is not a strategy of forgetting. Memorials and museums exhibit and also celebrate that which a society must forget to be able to continue existing.

Keywords: Memory. Memorials. Holocaust. Objects.

\footnotetext{
1 Ce texte est une version remaniée de l'article «La peine des hommes est-elle objet d'histoire ? Représentations et historicisations de l'holocauste", publié dans le Bulletin de la Fondation d'Auschwitz/Driemaandelijks tijdschrift van de Auschwitz stichting, Bruxelles, Belgique, 97, octobredécembre 2007, pp. 39-49 et de ses développements dans Montrer les violences extrêmes. Théoriser, créer, historiciser, muséographier, Annette Becker et Octave Debary (dir.), Paris, 2012, Créaphis, 350p.

${ }^{2}$ Anthropologue, Maître de conférences à l'Université Paris Descartes, Sorbonne Paris Cité. Membre du laboratoire de recherche du Lahic (EHESS-CNRS).
} 
Résumé: Cet article interroge la notion de musée et la relation entre histoire et mémoire à partir du difficile travail d'historicisation des objets dans les musées. Cette approche est une tentative d'étudier les mémoriaux et le processus de mémoire d'un point de vue anthropologique. Les débats qui entourent la représentation de l'holocauste, ainsi que le travail de l'artiste contemporain Jochen Gerz, servent de fil conducteur à cette réflexion. Je cherche ainsi à comprendre dans quelle mesure le devoir de mémoire n'est pas une stratégie d'oubli. Les mémoriaux et les musées exposent et célèbrent aussi ce qu'une société doit oublier pour exister.

Mots clés: Mémoire. Mémoriaux. Holocauste. Objets.

Avril 2004. Reprenant la traversée du Mall de Washington (DEBARY, 2004), je découvre le Musée Mémorial de l'Holocauste (E.T LINENTHAL, 1995 ; WEINBERG, ELIELI, 1995). Face au récit des camps de la mort de la seconde guerre mondiale, mon émotion se traduit par une incapacité à en dire quelque chose, dire quelque chose de cette histoire autant que de mon rapport à cette histoire. L'exposition se termine par une "salle du souvenir", une salle sans objet exposé. Nous sommes invités à nous asseoir, à nous souvenir silencieusement du drame ou à prier. Quelques jours plus tard, un paradoxe se formule : ce type de muséographie, dont la force repose sur l'émotion qu'elle provoque, conduit à rendre silencieux le visiteur. La conscience collective s'éveillerait dans une expérience intérieure et individuelle, hors du langage. Quel est le statut de la mémoire dans une société qui propose de se souvenir de son histoire et de ses drames par le silence?

Ce texte souhaite aborder la notion de musée et la question des rapports entre histoire et mémoire à partir d'une réflexion sur le difficile travail d'historicisation des objets dans les musées. Dans cette perspective, les débats soulevés par la représentation de l'holocauste serviront de fil conducteur à ce propos. Depuis Auschwitz, la question d'une juste représentation de l'histoire s'est posée dans toute sa radicalité. Depuis, " le problème représentatif du présent au passé ", cette capacité " du discours historique à représenter le passé", que Paul Ricoeur appelle "la représentance " (2000, p.306), a renvoyé l'adéquation entre mémoire et histoire à sa limite: “un traitement historiographique de l'inacceptable est-il possible ?"(Ibid, p.428). Un des enjeux de cette question, que je pose ici à titre d'hypothèse, est le suivant: les débats sur l'impossibilité de représenter l'holocauste révèlent la difficulté d'une historicisation de la mémoire de ce drame. Représenter l'holocauste, c'est en faire l'histoire, c'est historiciser la mémoire d'un inacceptable, qui est pourtant advenu. 
II s'agit de définir, autrement dit de penser, l'impensable, un événement historique au seuil, à la limite de ce qui appartient à l'histoire. Comment l'histoire peut-elle penser et représenter ce qui lui échappe et qui, dans le cas de la destruction des hommes euxmêmes, signifie la fin de toute histoire possible ?

Primo Levi dans Si c'est un homme a témoigné de son expérience concentrationnaire. Dans ce livre, rédigé entre décembre 1945 et janvier 1947, il explique ce besoin d'écriture comme "un besoin élémentaire" de "libération intérieure" (1987, p.8). Dans l'appendice de 1976, Levi se qualifie "d'écrivain témoin" (Ibid, p.214), d'homme à qui il revient de vivre pour raconter, de vivre pour témoigner. Un peu plus loin, on lui demande s'il est retourné à Auschwitz après la libération? Retourné sur les lieux en 1965, sa réponse est la suivante: "Il y a un musée où sont exposés de pitoyables vestiges, des tonnes de cheveux humains, des centaines de milliers de lunettes, des peignes, des blaireaux, des poupées, des chaussures d'enfants, mais cela reste un musée, quelque chose de figé, de réordonné, d'artificiel. Le camp tout entier m'a fait l'effet d'un musée" (Ibid, p.199). Par contre, là où il n'y a eu ni conservation, ni restauration, là où il n'y a pas de musée - à Birkenau-, on se rapproche d'un souvenir véritable. En ce sens, la non-conservation et l'abandon permettent de dire quelque chose des camps de la mort: "Là rien n'a changé, il y avait de la boue, et il y en a encore... là, rien n'a été enjolivé" (Ibid, p.200). Le musée d'Auschwitz rate son objet: impossible identification, impossible reconstitution de l'inhumain. Pourtant, Primo Levi en appelle au "devoir de mémoire" (1995). Le témoignage, comme récit, prend la forme d'un plaidoyer contre l'oubli. La mémoire est invoquée comme un devoir, une dette, devant le tribunal de l'histoire (TODOROV, 1998, p.12). Il s'agit, après coup, de repenser et de réconcilier ce qui s'est rompu.

S'interrogeant sur la possibilité pour l'historien de raconter et de montrer la souffrance des hommes, Philippe Braunstein s'est demandé si "la peine des hommes pouvait être un objet d'histoire" (2003, p.10)? Je propose de prolonger ce questionnement en reformulant l'un des enjeux qu'il sous-tend: dans quelle mesure les faits historiques peuvent s'établir sur la base d'un objet à proprement dit? La question doit ici s'entendre dans sa résonance muséographique. Quels types de musées et quels types d'objets peuvent raconter l'histoire? Cette difficulté de la mémoire à accueillir et à dire l'histoire est au cœur de la problématique muséale et historienne. La mémoire, comme le musée, n'est pas le lieu de l'histoire retrouvée, vérifiée. C'est au sein de cette problématique - de cette difficile réconciliation de I'histoire avec la mémoire- que s'inscrit la différence entre une mémoire apaisée et une mémoire falsifiée. S'ouvre ici la question politique et éthique d'une définition du juste souvenir et d'une juste mémoire: comment une société traite-elle de son passé? Le travail de mémoire s'inscrit 
dans la construction de ce qu'avec François Hartog on peut appeler des "régimes d'historicité" (2003) qui structurent et ordonnent des rapports au temps qu'un présent pose en miroir de sa définition du passé et du futur. Dans les musées, la caractéristique de ce travail de mémoire repose sur le déplacement de l'inscription de l'histoire des hommes vers les objets. On tend à faire de la mémoire un objet qui s'expose. Que I'histoire puisse ou non se réduire à un objet, l'opération de mise en passé de l'histoire, comme l'opération historiographique elle-même, construit l'expérience humaine sur la base d'un objet. Elle transforme l'absence en passé en séparant l'expérience de I'histoire. Cette opération est constitutive d'une science humaine qui fait de l'histoire une écriture de la séparation (DE CERTEAU (1975) 2002, p.138-142).

\section{OBJETS ET MÉMOIRES}

Le rapport entre histoire et mémoire peut-il être éclairé du lien qu'entretiennent les objets avec la mémoire (DEBARY; TURGEON, 2007)? Pour développer cette question, je me référerai à deux exemples empruntés à l'artiste allemand Jochen Gerz. Né à Berlin en 1940, Gerz vit et travaille en Irlande depuis 2008. Son travail est classé comme relevant de l'"art conceptuel". Cet adjectif maladroit tente de qualifier le traitement que l'artiste réserve à la matérialité de ses œuvres, la déjouant jusqu'à intégrer dans leur réalisation, le principe même de leur disparition. L'immatérialité de certaines de ses créations hisse le rapport entre le visible et l'invisible jusqu'au paradoxe qui amène l'artiste à définir l'art comme ce qui "donne à voir" (1996, p.165). L'art donnant également à voir ce qui échappe au regard.

Le premier exemple de son travail s'intitule Exit. Le projet Dachau (1972/1974). Pour Gerz, comme pour Primo Levi, le langage muséal est marqué d'un échec, voire d'une usurpation quant à sa faculté de représenter la complexité de l'histoire. Le musée conduit ainsi à "banaliser" (1994a, p.84) la violence de l'histoire. En réponse, son installation reconduit cette expérience d'une incorporation silencieuse de la violence, désignée comme violence muséale. Lors de la première biennale de Berlin en 1974, Gerz installe dans une salle sombre 20 tables disposées en deux rangées de 10, chacune avec sa chaise. Sur chaque table, un dossier contenant les photographies. Des lampes fixées au-dessus des tables dispensent un éclairage réduit. Une bande sonore ininterrompue reproduit le bruit de la respiration d'une personne en train de courir. Ce son est parfois recouvert par celui des frappes de deux machines à écrire. Cette installation part de l'idée que l'histoire relève des faits et que la culture repose sur une représentation de l'histoire ${ }^{3}$. Dans ce décalage, dans cette différence, les musées

\footnotetext{
${ }^{3}$ Voir les notes sur le projet Dachau (1974), reprises in Jochen Gerz (1994) : 84-85.
} 
inscrivent leur présence et proposent de penser l'histoire. Mais le langage muséal oublie ce décalage, il expose la violence de l'histoire avec une banalité toute aussi violente. Reconduire l'expérience de l'acceptation des ordres et des contraintes des visiteurs. Photographie du «règlement intérieur du musée»: il est "formellement interdit de fumer, d'amener des chiens, de détériorer les objets, d'amener des enfants non accompagnés, de faire du bruit... $»^{4}$. Gerz photographie le dispositif signalétique: "Sortie», "Entrée», une flèche qui indique la direction pour les «Hommes», celle pour les «Femmes» (toilettes). "Non recommandé aux visiteurs en dessous de l'âge de 13 ans», "prière de ne pas écrire sur le mur». Une porte fermée: "Administration». Les matériaux rassemblés pour le Projet Dachau surexposent la structure linguistique du musée -celle qui oblitère et prolonge la violence du passé des lieux- et celle du camp. Une porte fermée, "aucun accès». Comment sortir? Des listes de dizaines de noms. Nom, prénom, origine: Per Andersson, Sweden, Peter Rosberg, Sweden, Mr et Miss Stephan Elomli, USA,...... il s'agit du livre d'or. Le livre est près d'un mur en haut duquel on aperçoit en lettres capitales «AUSGANG»/«SORTIE». Les visiteurs de I'histoire inscrivent leurs noms, ultime étape avant leur sortie (Ibid, p.115-116). Comme I'explique Irit Rogoff : «The museum which commemorates the camp, (...), is shown by Gerz in his project to function along not unsimular guidelines in which the public is marshalled, classified and meagrely informed through a sign system of authoritative regulation witch equally ends with an exit» (1993, p.42). Gerz interroge la structure de la syntaxe muséale en tant que structure de classement, d'ordonnancement et de violence : «l'organisation linguistique du musée de Dachau et l'organisation linguistique du camp de concentration ne s'opposent qu'en apparence ; elles se complètent plutôt, chacune en tant que projet de l'autre» (1994a, p.85).

Comment raconter Dachau ici et maintenant? Comment raconter la Shoah? Comment sortir de Dachau? II fallait transformer ce souvenir impossible, transformer le camp en musée pour le visiter. S'y promener, signer son nom sur un livre d'or, suivre le sens de la visite jusqu'au panneau «Sortie». Oublier que les gens ont suivi l'écriteau «Brausebad» («douche») à l'entrée des chambres à gaz, comme dernier message de la langue. Il fallait oublier cette ressemblance, la ressemblance entre le musée et le camp. Le musée est davantage un espace de relégation que de monstration. Dédié à l'exposition de ce qui n'est plus, le passé. Montrer ce qui n'est plus à partir de ce qui reste, un reste irréductible que l'on conserve, expose et, en même temps que l'on enferme, cache (claquemurer, calfeutrer). Donnant des lieux, des tombes à l'histoire, en

${ }^{4}$ Jochen Gerz, Francis Lévy (1978), voir pp. 44-45, 47, 53, 54-55, 56-57. 
séparant le présent du passé. La politique mémorielle de la pierre est travaillée par l’oubli.

Le second exemple emprunté à Gerz s'intitule Le Monument de Harburg contre le Fascisme (Das Harburger Mahnmal gegen Faschismus) réalisé en 1986 par l'artiste et sa femme, Esther Shalev-Gerz, en Allemagne à Harburg (banlieue de Hambourg). A sa création, le Monument se présente sous la forme d'une colonne de douze mètres de hauteur recouverte de plaques de plomb vierge. Gerz a invité les habitants à les signer, à signer ce monument contre le fascisme. Le dispositif permet à la colonne de s'enfoncer progressivement dans le sol de deux mètres par année. Plus de 60000 personnes sont venues signer le monument. Des gens signaient leurs noms, d'autres les rayaient. Certains sont revenus réinscrire leurs noms. Comme l'explique Gerz: "Nous avons été surpris par la violence du public. Toutes les signatures étaient immédiatement grattées et rayées par des insultes. Des gens ont tiré sur le monument, d'autres ont utilisés des scies, des couteaux." (GERZ, 1994b, p.45). "Peu à peu, les signatures sont devenues quelques mots puis des phrases" (ESTHER SHALEV; GERZ, 1994b, p.27). On pouvait lire "Nous sommes contre le fascisme", ailleurs, "Nous sommes pour le fascisme". En 1993, le temps passant, le dispositif d'enterrement est arrivé à terme, la colonne a disparu. Depuis, cette véritable pétition contre le fascisme appelle le souvenir par son contraire: la disparition et l'oubli. Les signataires du monument trouvent dans la disparition de la colonne le besoin de raconter son histoire, l'histoire du monument se transmet à ceux qui ne l'ont pas connu (à ceux qui n'étaient pas là -pour voir le monument). Ayant signés de leurs noms et après avoir été littéralement enterrés vivants, les signataires établissent un lien entre eux et l'histoire du monument. Cette œuvre, en provoquant sa propre disparition fait œuvre de mémoire. Les gens racontent l'histoire du monument, le souvenir d'une mémoire invisible. A l'inverse, les mémoriaux classiques sont voués à l'oubli et avec eux, I'histoire qu'ils sont censés rappeler. Chacun d'eux pense et se souvient pour nous. II faut aller les voir pour se souvenir. Le monument délègue le souvenir à la mémoire de ses pierres, "il concourt ainsi au refoulement qu'il favorise et bénit ; un peu comme si, à l'instar de ces veilleurs qui parcouraient jadis les rues des villes durant la nuit répétant "dormez braves gens !", tout monument disait à chaque citoyen, "oubliez, je me souviens" (WAJCMAN, 1998, p.201). Ce refus de la fonction monumentale classique chez Gerz s'adresse, selon ses mots, "aux gens qui ont longtemps dit on n'a rien vu, on n'était pas là ", l'objet répond: "je ne suis pas là non plus" (1996, p.157). Ce qui reste d'apparent à Harburg, c'est une inscription à côté du Monument dont la dernière phrase est: "Le jour où elle aura disparu, l'emplacement du monument de Harburg sera vide. Car rien ne peut se dresser à notre place contre l'injustice ". C'est dans cette 
différence entre l'histoire et la mémoire que le monument est invité à tenir lieu (à notre place) de mémoire. Le lieu de mémoire est aussi ce qui se substitue à l'histoire. Gerz plaide pour une poétique de la disparition de l'objet en sacrifiant sa visibilité. II s'agit de rétablir l'histoire par le récit, le manque et l'absence devenant les modalités d'une mise en présence de l'histoire: quelque chose a lieu, quelque chose pose question. Dans cette lutte de la mémoire, l'anti-monument ("Gegen-Denkmal" ou "countermonument") de Gerz se construit comme souvenir de la disparition: "All the remains, then, is the memory of the monument, an afterimage projected onto the landscape by the rememberer. The best monument, in Gerz's view, may be no monument at all, but only the memory of absent monument " (YOUNG, 1993, p.32). L'invisibilité de la colonne signifie le refus d'une éthique de la mémoire fondée sur le voir au profit d'une éthique du savoir. Le témoin et le responsable de l'histoire n'est pas celui qui voit mais celui qui sait ou doit savoir même si l'histoire, comme la colonne, reste cachée, hors de sa vue. Christo met en œuvre des dispositifs comparables dans son art d'emballer les monuments. Confisquant leur visibilité aux monuments, il questionne le rôle que l'on fait jouer à une mémoire monumentale pétrifiée qui refoule I'historicité, "un passé sous surveillance" que Christo préfère "ficeler" pour reprendre l'expression de Régine Robin (In GERZ, 1994b, p.55). De la même manière, Gerz congédie l'objet en tant qu'objet, le monument en tant que monument, comme tenant lieu de mémoire: "les objets, au fond, n'existent pas", dit-il (1994b, p.11).

\section{PENSER L'HOLOCAUSTE}

Comment les débats autour de la représentation de l'holocauste s'inscrivent dans ce questionnement? Je ne m'attarderai pas sur les enjeux d'une politique de la mémoire liée à ce drame dont la littérature abondante et parfois polémique dépasse le cadre de mon propos.

En reprenant la distinction qu'opère Maurice Halbwachs dans La mémoire collective ((1950), 1997) entre mémoire et conscience historique, Peter Novick a défendu l'idée selon laquelle le souvenir de l'holocauste est aujourd'hui marqué par le primat accordé à la mémoire sur la conscience historique. La conscience historique porte sur l'historicité des événements, sur les raisons historiques qui expliquent le caractère -contemporain- d'un événement. La mémoire, elle, n'inscrit pas sa présence dans le passage du temps, elle déhistoricise le souvenir et, selon Novick, "nie ainsi l'appartenance au passé de ses objets en insistant sur leur présence continue" (1999 2001, p.10). A l'opposé d'une recherche et d'une analyse des conditions historiques de son apparition et de sa raison, le souvenir de l'holocauste porte sur une mémoire qui vient dire et montrer l'événement, plus que sur des recherches d'explications de 
l'événement. Selon Yannis Thanassekos, ce type de rapport à l'histoire est typique d'une muséographie postmoderne qui "neutralise le passé historique" sous figure de le raconter. Cette tendance caractérise une société du spectacle dans laquelle «la surdétermination esthétique des enjeux muséologiques (...)expulse le référentiel historique et mémoriel(...)» (1996, p.33-34). Comme si avant de comprendre une chose -liée à la disparition et au drame- il s'agissait de signifier l'événement de la chose. A la question, comment cela a-t-il été possible, on répond souvent cela a eu lieu.

L'affirmation historique du drame, en se soustrayant à sa raison historique, échappe à son historicité, à "sa conscience historique". Resurgit ici la difficulté de penser l'holocauste. Evénement dont l'incommensurabilité dans l'échelle de l'horreur, fonde une unicité souvent synonyme de l'impossibilité de sa représentation. L'impossible comparaison de ce drame à d'autres, son unicité, viennent fonder le refus de sa représentation. Si le secret de la "vérité" de ce drame "réside dans le silence" et "défie toute connaissance et toute description" selon Elie Wiesel (1994, p.123), alors il n'y a qu'un pas de plus à faire pour rendre la tentative "de compréhension rationnelle de l'holocauste équivalente à sa négation" pour reprendre les mots de Norman Finkelstein (2001, p.47). Entendons-nous bien sur l'enjeu de ce débat, évitons donc toute ambiguïté inhérente à la manière dont cette question de la représentation de I'holocauste se pose trop souvent, ou plutôt refuse de se poser autrement. C'est le refus de son historicisation au profit de sa commémoration que je tente de questionner. Pour le dire différemment, je me demande si le refus de représenter ce drame ne conduit pas à le rendre silencieux, à rendre silencieux son souvenir. C'est précisément la tentative de compréhension de ce drame, sa compréhension historique, qu'a entrepris courageusement Raul Hilberg. Son œuvre est consacrée à la recherche historique des fondements et des mécanismes de l'entreprise bureaucratique de destruction menée par les nazis (1988 et 2001). Hilberg tente ainsi de construire une mémoire historique de l'holocauste. Pour cette raison et comme il l'explique, il opère un renversement du point de vue classique en s'attachant à comprendre l'inacceptable, autrement dit, le point de vue des bourreaux: “J'étais convaincu qu'il était impossible de saisir la pleine dimension de ce fait historique si l'on ne comprenait pas les mécanismes des actes des exécuteurs" (1996, p.57).

Ce renversement est central. Si l'on prend acte des mots de Hilberg, la compréhension de l'holocauste ne peut pas faire l'économie d'une compréhension des bourreaux. On peut penser que c'est cette difficulté qui a conduit à porter la mémoire de I'holocauste du côté des victimes. Comme le souligne Hilberg, "presque tous les monuments érigés aux Etats-Unis ou en Israël, qu'il s'agisse d'encyclopédies, d'instituts ou de musées, ont pour pierre angulaire l'attention portée à la victime et non à 
l'exterminateur"(Ibid, p.123). Le processus de dé historicisation ne commence-t-il pas aussi ici ? Le refus d'historiciser la mémoire de l'holocauste du point de vue des bourreaux ne peut-il pas s'expliquer par le refus de donner un visage humain aux coupables? C'est là le centre du débat qui nous préoccupe, l'inhumanité de I'holocauste serait venue fonder la difficulté de le penser et de le représenter. Cette tendance a pour conséquence de conduire au silence de la mémoire de ce drame. L'importance accordée à une définition de l'holocauste attachée à la perte et non à son explication est exemplaire dans le cas du Musée Mémorial de l'holocauste de Washington. Le geste inaugural d'entrée dans l'exposition -et donc dans l'histoireconsiste à distribuer et assigner à chaque visiteur la carte d'identité d'une victime (et non d'un bourreau). Ce parti pris du Musée est fondé sur l'idée selon laquelle : «To facilitate the emotional identification process, it was essential that visitors perceive the victims as humaun individuals rather than as a mass of dehumanized (...) " (WEINBERG ; ELIELI, 1995, p.71). La connaissance de I'histoire est soumise à un processus d'identification individuelle aux victimes en réponse à une histoire qui les a détruites massivement et anonymement. Si Auschwitz reste un "cimetière sans tombe», la mémoire du camp ne peut s'y réduire car elle est aussi marquée par l'histoire des bourreaux. Hilberg, lui, tente également de rétablir les noms, les logiques et les intentions des bourreaux. Dans ce mouvement, il ne nie pas, ni n'affaiblit, leur culpabilité, mais tente d'établir leur responsabilité au regard de leur historicité. La difficulté de l'entreprise menée par Hilberg explique la réception et la publication problématiques de son travail à ses débuts comme il le racontera dans son ouvrage $L a$ politique de la mémoire (1996). En rétablissant cette mémoire historique de I'holocauste, comme entreprise de destruction intentionnelle, Hilberg redonne la parole à l'histoire. L'unicité de cette catastrophe peut alors s'énoncer, sans concession, dans l'unicité de son historicité.

De l'expérience de Primo Levi retournant à Auschwitz, on pourrait dire que ce n'est pas tant la mémoire (pour le coup, soumise à un véritable travail d'écriture et de mise en récit) qui est absente mais l'histoire qui est toujours présente. La question de la "représentance" n'a pas lieu, elle ne trouve pas de lieu où s'inscrire. Cette impossible mise à distance de l'histoire et de mise en passé de l'expérience concentrationnaire lui fera dire dans Les Naufragés et les Rescapés, le difficile cheminement de la mémoire face au problème d'avoir à "raconter sa propre mort" (1989, p.83). C'est en ce sens que le musée d'Auschwitz rate son objet. En refusant de reconnaître à ce musée un juste souvenir du drame, Levi vient aussi dire l'impossible constitution de cette histoire comme objet de mémoire. Quels mots lui viennent pour qualifier cette visite du musée d'Auschwitz. Ecoutons-les à nouveau : "Il y a un musée où sont exposés de pitoyables 
vestiges, des tonnes de cheveux humains, des centaines de milliers de lunettes, des peignes, des blaireaux, des poupées, des chaussures d'enfants, mais cela reste un musée, quelque chose de figé, de réordonné, d'artificiel. Le camp tout entier m'a fait l'effet d'un musée" (1987, p.199). Dans cette dernière phrase, le qualificatif “ musée " sert à définir toute expérience ratée de la rencontre avec l'histoire. Le paradoxe muséal se précise: si les musées prétendent raconter l'histoire en exposant des objets, ils concourent tout autant à la construction d'une mémoire déhistoricisée, "figée", "réordonnée", ou "enjolivée" pour reprendre les mots de Levi.

Les musées refusent souvent la discontinuité du temps en réduisant le travail de mémoire à une présence cumulative de ses objets. Leur présence suture le vide et la perte en réparant, en collectionnant des objets comme autant de dénis du temps qui passe. Collectionner ce déni et l'enfermer dans l'espace clos du musée. Le conservateur, quand il n'est qu'un simple collectionneur, se trouve condamné à la répétition de son geste d'acquisition parce qu'à chaque fois il rate son objet. L'objet demeure manqué, manquant. La collection multiplie davantage les signes de l'absence que les objets eux-mêmes. Mon propos ne vise pas à récuser l'objet mais à inviter à réfléchir aux modalités d'accueil de son historicité. Se pose ainsi la question de savoir comment accueillir l'histoire comme ce qui et ce que contient l'objet. Cette question s'inscrit pleinement dans cette "inéluctable scission du voir" dont parle DidiHuberman. Scission qui fait que "ce que nous voyons ne vaut -ne vit- à nos yeux que par ce qui nous regarde" (1992, p.9). C'est dans cet entre deux qu'habitent l'art de Gerz et certains musées. II s'agit d'arriver à amener le spectateur à questionner ce qui, dans sa rencontre avec l'objet, le regarde. Qu'est-ce qui nous regarde dans l'objet de musée, dans l'objet de mémoire?

Si la mémoire se conjugue toujours au présent, cet anachronisme du souvenir ouvre au manque né de la rencontre avec l'objet. L'objet dit quelque chose en même temps qu'il convoque ce qui lui échappe. C'est ce travail de la mémoire que vise Gerz et qu'il appelle la présence d'un "passé négatif”(1994b, p.8). L'histoire des autres inatteignable en tant que passée- s'atteint par sa négativité photographique, comme mémoire revisitée au présent. Par ce pouvoir, l'objet - des autres- peut devenir "objet d'histoire" -commune- pour reprendre la question de Philippe Braunstein. Un objet d'histoire, un objet de questions (HAINARD, 1983, p.2005) et de recherches (CHIVA, 1997): un objet dont la mise en récit s'offre au muséographe et à son écriture. C'est dans la limite de la matérialité de l'objet -cet objet impossible- que surgit positivement son immatérialité, une immatérialité que je me propose de définir par un mot: I'histoire entendue comme "reste nocturne" (DIDI-HUBERMAN, 1992, p.145). Un reste 
nocturne dont le réveil à la conscience donne toute sa valeur à la notion de travail de mémoire.

\section{BIBLIOGRAPHIE}

BECKER Annette; DEBARY, Octave (dir.). Montrer les violences extrêmes. Théoriser, créer, historiciser, muséographier. Paris: Créaphis, 2012.

BRAUNSTEIN, Philippe. Travail et entreprise au Moyen Âge. Bruxelles: De Boeck, 2003.

CHIVA, Isac. L'objet d'exposition: témoigner pour les uns, signifier pour les autres. In Hainard, J. ; Kaehr, R. (dir.). Dire les autres. Hommages rendus à Pierre Centlivres. Lausanne: Payot, 1997, p.359-363.

DE CERTEAU, Michel. L'écriture de l’histoire. Paris: Gallimard, (1975) 2002.

DEBARY, Octave. Lettre de Washington. Urbanisme, 334, janv-fév. 2004, p.29-30.

DEBARY, Octave, Turgeon, Laurier (dir). Objets \& Mémoires. Paris-Québec: Maison des sciences de l'Homme et Presse de l'Université Laval, 2007.

DIDI-HUBERMAN, Georges. Ce qui nous regarde, ce que nous voyons. Paris: Minuit, 1992.

FINKELSTEIN, Norman G.. L'industrie de l'Holocauste: réflexions sur l'exploitation de la souffrance des Juifs. Paris: La Fabrique, (2000) 2001.

GERZ, Jochen; LÉVY, Francis. Exit, Das Dachau-Projekt. Frankfurt: Verlarg Roter Stern, 1978.

GERZ, Jochen. De l’art: textes depuis 1969. Paris: Ensb-a, 1994 (a).

------ Fragments. Strasbourg: LimeLight/Ciné-Fils, 1994 (b).

------ La question secrète. Paris: Actes Sud, 1996.

GERZ, Jochen; LÉVY, Francis. Exit, Das Dachau-Projekt. Frankfurt: Verlarg Roter Stern, 1978. HAINARD, Jacques. Le musée comme enjeu. In Quels musées pour quelles fins aujourd'hui?. Séminaires de l'Ecole du Louvre. Paris: La Documentation française, 1983, p.75-83.

Du musée spectacle à la muséographie de la rupture. In GONSETH, Olivier; HAINARD, Jacques; Kaehr, Roland(dir.). Cent ans d’ethnographie. Neuchâtel: Musée d'ethnographie de Neuchâtel, 2005, p.367-373.

HALBWACHS, Maurice. La mémoire collective. Paris: Albin Michel, (1950) 1997. 
HARTOG, François. Régimes d'historicité, présentisme et expériences du temps. Paris: Seuil, 2003.

HILBERG, Raul. La destruction des juifs d’Europe. Paris: Fayard, (1961) 1988.

----- La politique de la mémoire. Paris: Gallimard, (1994) 1996.

------ Holocauste : les sources de l'histoire. Paris: Gallimard, (2001) 2001.

LEVI, Primo. Si c'est un homme. Paris: Julliard, (1947) 1987.

----- Les naufragés et les rescapés. Paris: Gallimard, (1986) 1989.

------ Le devoir de mémoire. Paris: Mille et une nuite, (1989)1995.

LINENTHAL, T. Edward. Preserving Memory. New York: Penguin Book, 1995.

NOVICK, Peter, L'Holocauste dans la vie américaine. Paris: Gallimard, (1999) 2001.

RICOEUR, Paul. La mémoire, l'histoire, l'oubli. Paris: Seuil, 2000.

ROGOFF, Irit. That obscure object of desire. Reflections on loss, erasure and the politics of commemoration. In GERZ, Jochen. 2146 Stones, Memorial against Racism Saarbrücken, 1993.

THANASSEKOS, Yannis. Musée, histoire, mémoire et identité dans l'ère postmoderne. In The contemporary museum within the postmoderne era/Le musée contemporain dans l'ère postmoderne. ROZANIS, Stephanos; THANASSEKOS, Yannis (dir). Athènes-Bruxelles: The Jewish Museum of Greece, la Fondation d'Auschwitz, 1996, p.23-45.

TODOROV, Tzvetan. Les abus de la mémoire. Paris: Arléa, 1998.

WAJCMAN, Gérard. L'objet du siècle. Paris: Verdier, 1998.

WEINBERG, Jeshajahu, Elieli Rina. The Holocaust Memorial Museum in Washington. New York: Rizzoli, 1995.

WIESEL, Elie. Tous les fleuves vont à la mer. Paris: Seuil, 1994.

YOUNG, James E.. The Texture of Memory. New Haven and London: Yale University Press, 1993. 